\title{
Avaliação da cultura de segurança no centro cirúrgico: Uma revisão integrativa
}

\author{
Assessment of the safety culture in the surgical center: An integrative review \\ Evaluación de la cultura de seguridad en el quirófano: Una revisión integradora
}

Recebido: 12/07/2021 | Revisado: 19/07/2021 | Aceito: 26/07/2021 | Publicado: 02/08/2021

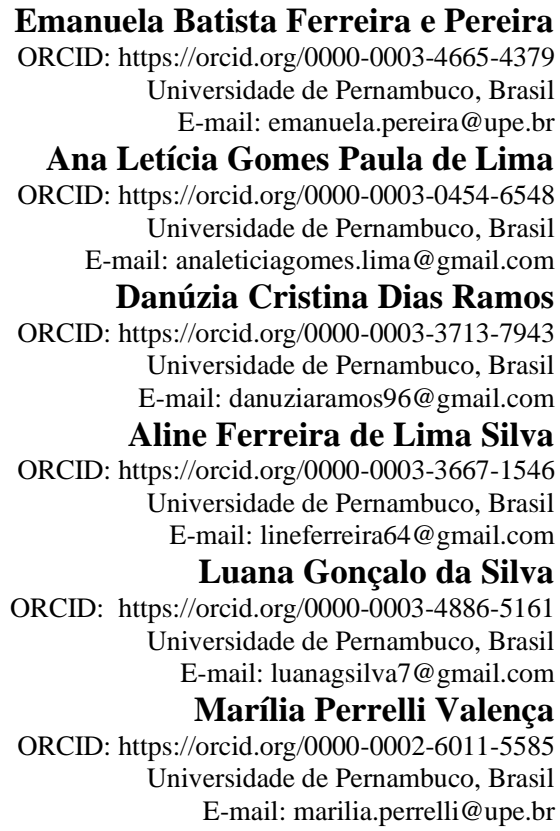

\section{Resumo}

Objetivo: Conhecer a cultura de segurança encontrada no centro cirúrgico. Metodologia: Trata-se de uma revisão integrativa da literatura que utilizou como guia a metodologia PRISMA nas publicações no período dos últimos cinco anos (2015 a 2020) nas bases de dados MEDLINE, PUBMED, LILACS e BDENF - Enfermagem nos idiomas inglês, português, espanhol e textos disponíveis na íntegra. Para extração dos dados dos artigos elaborou-se um instrumento contendo as seguintes variantes: título, autor, ano, base de dados, cruzamento de descritores, período, tipo de estudo, população, amostra, local de estudo, adesão da segurança, avaliação da segurança do paciente, contribuição do estudo para equipe de saúde. Resultados: Foram selecionados 5 artigos após exclusão por fuga ao tema, indisponibilidade de texto na íntegra e que respondiam à questão norteadora: "Qual cultura de segurança presente no centro cirúrgico?". Após análise criteriosa, observou-se que a cultura de segurança no ambiente cirúrgico é considerada um elemento chave para reduzir eventos adversos e visa garantir que equipes cirúrgicas desenvolvam os protocolos de segurança com criticidade. Conclusão: As Instituições de saúde cada vez mais utilizam como estratégia a avaliação da cultura de segurança, para garantir uma assistência de qualidade, com ausência de erros e eventos adversos.

Palavras-chave: Centros cirúrgicos; Segurança do paciente; Cultura organizacional.

\begin{abstract}
Objective: To understand the safety culture found in the operating room. Methodology: This is an integrative literature review that used the PRISMA methodology as a guide in publications over the last five years (2015 to 2020) in the MEDLINE, PUBMED, LILACS and BDENF - Nursing in English, Portuguese, Spanish and texts available in full. To extract data from the articles, an instrument was developed containing the following variants: title, author, year, database, crossing of descriptors, period, type of study, population, sample, study site, safety adherence, evaluation of patient safety, study contribution to the health team. Results: Five articles were selected after exclusion due to escape from the topic, unavailability of the full text and that answered the guiding question: "What is the safety culture present in the operating room?". After careful analysis, it was observed that the safety culture in the surgical environment is considered a key element to reduce adverse events and aims to ensure that surgical teams develop safety protocols critically. Conclusion: Health institutions increasingly use the evaluation of the safety culture as a strategy to ensure quality care, with the absence of errors and adverse events.
\end{abstract}

Keywords: Surgical centers; Patient safety; Organizational culture. 


\begin{abstract}
Resumen
Objetivo: Comprender la cultura de seguridad que se encuentra en el quirófano. Metodología: Se trata de una revisión integradora de la literatura que utilizó la metodología PRISMA como guía en las publicaciones de los últimos cinco años (2015 a 2020) en MEDLINE, PUBMED, LILACS y BDENF - Enfermería en inglés, portugués, español y textos disponibles en su totalidad. Para extraer datos de los artículos, se desarrolló un instrumento que contiene las siguientes variantes: título, autor, año, base de datos, cruce de descriptores, período, tipo de estudio, población, muestra, sitio de estudio, adherencia a la seguridad, evaluación de la seguridad del paciente, estudio contribución al equipo de salud. Resultados: Se seleccionaron cinco artículos luego de la exclusión por escape del tema, falta de disponibilidad del texto completo y que respondieron a la pregunta orientadora: “¿Cuál es la cultura de seguridad presente en el quirófano?”. Después de un análisis cuidadoso, se observó que la cultura de seguridad en el entorno quirúrgico se considera un elemento clave para reducir los eventos adversos y tiene como objetivo garantizar que los equipos quirúrgicos desarrollen protocolos de seguridad de manera crítica. Conclusión: Las instituciones de salud utilizan cada vez más la evaluación de la cultura de seguridad como estrategia para asegurar una atención de calidad, sin errores y eventos adversos.
\end{abstract}

Palabras clave: Centros quirúrgicos; Seguridad del paciente; Cultura organizacional.

\title{
1. Introdução
}

Entre os anos de 460 a 370 a.C, Hipócrates postulava a seguinte máxima: Primum non nocere, que na tradução significa: primeiro não cause danos. Com o passar dos séculos o cuidado em saúde tornou-se cada vez mais complexo, juntamente com o risco para erros e eventos adversos. Diante disso, a Segurança do Paciente consiste na redução a um mínimo aceitável do risco de dano desnecessário associado ao processo de assistência à saúde (Brasil, 2014).

Nesse contexto, a Organização Mundial de Saúde (OMS), em 2004, impulsionada a reduzir os danos causados pela assistência em saúde, criou o programa Aliança Global para Segurança do Paciente, que busca incentivar práticas e estratégias voltadas para a segurança e assim consequentemente ampliar o desenvolvimento e a discussão em torno da temática (Wegner, et al., 2016).

No Brasil, ano de 2013, o Ministério da Saúde instituiu o Programa Nacional de Segurança do Paciente (PNSP) que visa promover e estimular iniciativas que fortaleçam o estabelecimento da cultura de segurança como estratégia para a garantia de cuidados em saúde livres de danos e erros. Além disso, buscou-se fortalecer uma cultura de segurança sem caráter punitivo, modificando a concepção de que a percepção dos erros é uma forma de instruir-se com as falhas e consequentemente proporcionar a adoção de práticas seguras com redução de incidentes (Dezordi \& Stumm, 2018; Heidmann et al., 2020).

Pode-se compreender a cultura de segurança como a união de diversos fatores, entre eles estão: atitudes, conhecimentos, percepções e práticas que podem ser individuais ou em grupo. A supervisão da cultura de segurança pode contribuir para promoção do trabalho em equipe e cuidados seguros ao longo da permanência do paciente no serviço através de seu envolvimento como gestor de segurança da instituição (Sanchis et al., 2020).

Para que seja incorporada em um serviço é necessária a força conjunta entre a gerência e os profissionais atuantes nos cenários de assistência ao paciente. Além do exposto, outros fatores, dentre eles, a satisfação no trabalho, que influencia diretamente a cultura de segurança e que pode estar relacionada ao número de ocorrência de eventos adversos. A avaliação da cultura de segurança permite, de forma direta, a identificação das fragilidades encontradas no serviço, e consequentemente, o investimento que será necessário para correção desta fragilidade (Sanchis et al., 2020).

Nesta perspectiva, para promover a cultura de segurança foi implementado o Programa Nacional de Segurança do Paciente (PNSP), que contempla os seguintes protocolos básicos: identificação do paciente; segurança na prescrição, uso e administração de medicamentos; cirurgia segura; prática de higiene das mãos em serviços de saúde e prevenção do risco de quedas e úlceras por pressão, que visam nortear as condutas dos profissionais na prevenção de erros associados aos cuidados em saúde (Serra et al., 2016).

Entre os protocolos básicos do PNSP destaca-se a cirurgia segura e na esfera hospitalar, o setor do Centro Cirúrgico 
(CC) proporciona cuidados intervencionistas ao paciente, buscando a recuperação ou melhora por meio do ato cirúrgico. Sua multifatorialidade é atribuída à quantidade de procedimentos complexos realizados, à relação entre os diversos profissionais que trabalham nesse meio e à pressão, pois apesar de seu impacto terapêutico o CC pode apresentar agravos associados à saúde do paciente, como complicações cirúrgicas, erros e mortes (Henriques et al., 2016).

Diante da preocupação com os erros e danos associados às práticas cirúrgicas o segundo Desafio Global para Segurança do Paciente, "Cirurgia Segura Salva vidas", reforça que práticas assistenciais seguras no ambiente cirúrgico reduz as mortes e complicações cirúrgicas, por meio da adoção do checklist ou Lista de Verificação Cirúrgica, como uma rotina universal que proporcionou alterações que refletem no trabalho em equipe, como mudanças nas atitudes por parte de todos os membros da equipe cirúrgica (Marique et al., 2015).

Nesta perspectiva, a adaptação dessas mudanças apresentadas no âmbito cirúrgico ao longo dos anos, refletem nas condutas que a equipe multiprofissional possui, sendo importante para a melhoria na qualidade da assistência, proporcionando assim, boas práticas em saúde. Diante do exposto, o presente estudo teve como objetivo realizar uma revisão integrativa para avaliar a cultura de segurança encontrada no centro cirúrgico.

\section{Metodologia}

Trata-se de uma revisão integrativa que utilizou a metodologia PRISMA como guia. Realizou-se uma busca eletrônica na Biblioteca Virtual em Saúde (BVS), nas bases de dados MEDLINE, PUBMED, LILACS e BDENF - Enfermagem, em fevereiro de 2020. Os descritores "Patient Safety", "Organizational Culture" e "Surgery Centers" foram utilizados como estratégia de busca para identificação dos artigos através de dois cruzamentos: "Organizational Culture and Surgery Centers", e "Patient Safety and Surgery Centers".

Os seguintes critérios de elegibilidade foram empregados: artigos completos em inglês, português e espanhol, publicados nos últimos cinco anos (2015-2020), com textos disponíveis na íntegra e que respondiam à pergunta norteadora desta pesquisa: qual a avaliação da cultura de segurança presente no centro cirúrgico?

Os artigos com duplicação entre as bases foram identificados. Após a leitura do título e resumo, aqueles que não atendiam aos critérios de elegibilidade, ou que se desviavam da temática abordada, foram excluídos. Nos casos em que a leitura do resumo era insuficiente para determinar a inclusão do artigo, o texto foi lido na íntegra; os demais foram selecionados e obtidos de forma integral para confirmação da elegibilidade.

Para extração dos dados dos artigos elaborou-se um instrumento próprio contendo as seguintes variantes: título, autor, ano, base de dados, cruzamento de descritores, período, tipo de estudo, população, amostra, local de estudo, adesão da segurança, avaliação da segurança do paciente, contribuição do estudo para equipe de saúde.

\section{Resultados}

Após a eliminação de 3 artigos duplicados, foram selecionados 333 artigos. Desses, 288 foram excluídos por título e resumo, e por estarem indisponíveis na íntegra. Restando 45 artigos rastreados, desse subtotal 40 foram excluídos por, após leitura de texto, fugirem ao tema, restando 5 artigos das seguintes bases de dados: 1 PUBMED, 2 MEDLINE, 1 na LILACS e 1 na BDENF - Enfermagem que após análise mais detalhadas foram incluídos na presente revisão integrativa (Figura 1). 
Figura 1 - Fluxograma da seleção de artigos, Recife, 2021.

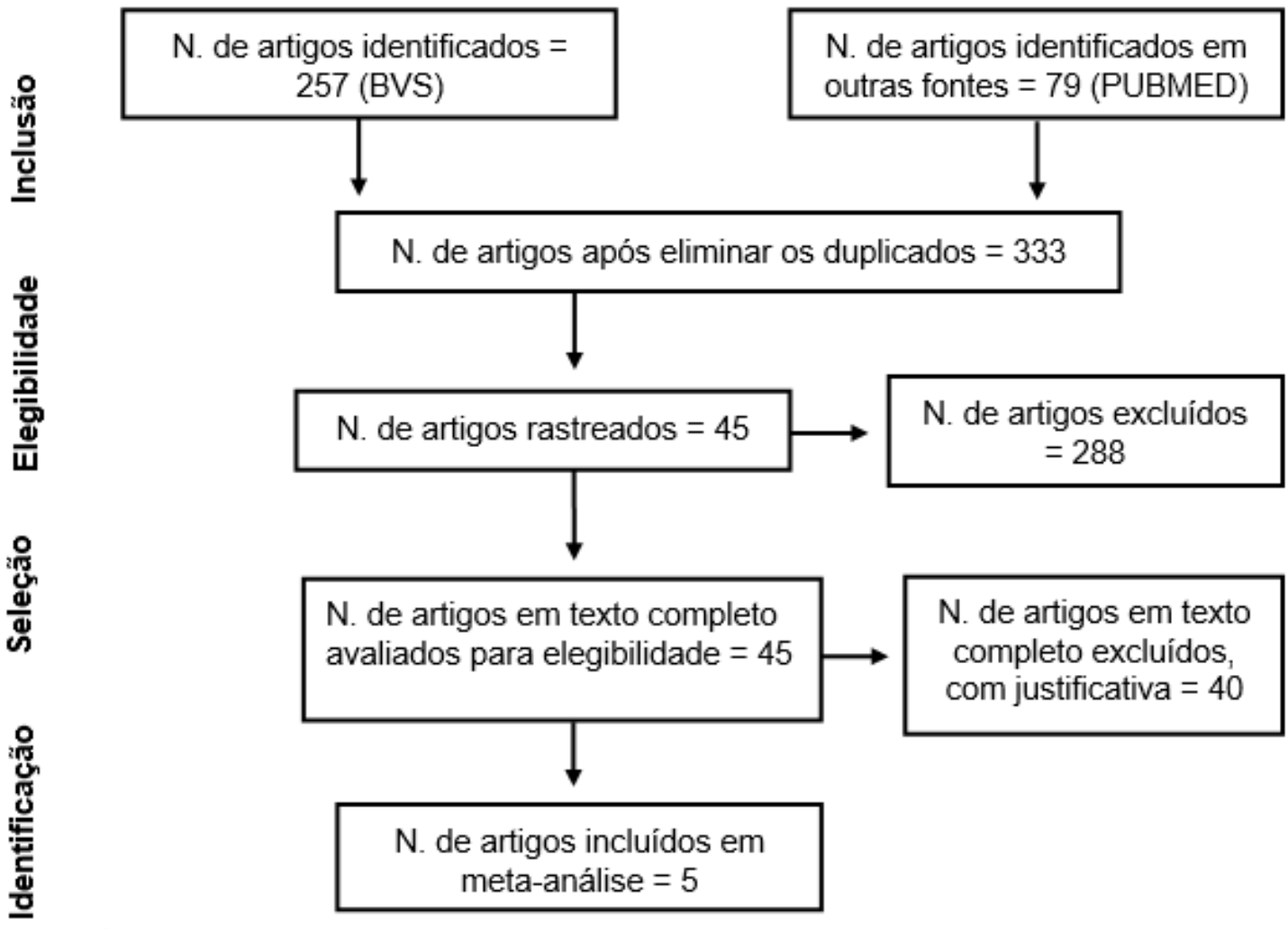

Fonte: Autoras (2020).

Quanto às características gerais, as publicações mais frequentes foram do ano de 2017 (40\%); as bases de dados que mais apareceram nas pesquisas foram MEDLINE e LILACS. O delineamento do tipo transversal foi predominante com $80 \%$. Em relação a população do estudo, foi observado uma predominância da equipe atuante no centro cirúrgico (C.C) com $80 \%$ (Quadro 1).

Conforme o seguimento da análise dos artigos selecionados evidenciou-se que o país com mais publicações de artigos foi o Brasil (60\%). Com relação ao tempo de duração das pesquisas, obteve média de 6 meses e 25 dias e em média foram 254,8 profissionais envolvidos nas pesquisas (Quadro 1). 
Quadro 1. Descrição das características dos artigos incluídos. Recife, 2020.

\begin{tabular}{|c|c|c|c|c|c|c|c|}
\hline Título do artigo & Autor/Ano & $\begin{array}{c}\text { Base de } \\
\text { Dados }\end{array}$ & País & Tipo de Estudo & $\begin{array}{c}\text { Amostra do } \\
\text { Estudo }\end{array}$ & $\begin{array}{c}\text { Duração } \\
\text { do Estudo }\end{array}$ & $\begin{array}{c}\text { População do } \\
\text { Estudo }\end{array}$ \\
\hline $\begin{array}{l}\text { Cultura de segurança } \\
\text { do paciente em um } \\
\text { centro cirúrgico: a } \\
\text { percepção } \\
\text { enfermagem }\end{array}$ & $\begin{array}{l}\text { Abreu, et al; } \\
2019 \text { (E1) }\end{array}$ & PUBMED & Brasil & Transversal & $\begin{array}{l}92 \\
\text { Profissionais }\end{array}$ & 6 Meses & $\begin{array}{l}\text { Enfermeiros } \\
\text { no C.C }\end{array}$ \\
\hline $\begin{array}{l}\text { Comparing safety } \\
\text { climate for nurses } \\
\text { working in operating } \\
\text { theatres, critical care } \\
\text { and ward areas in the } \\
\text { UK: a mixed methods } \\
\text { study. }\end{array}$ & $\begin{array}{l}\text { Tarling, et al. } \\
2017 \\
\text { (E2) }\end{array}$ & MEDLINE & Europa & Transversal & 319 & - & Enfermeiros \\
\hline $\begin{array}{l}\text { Clima de segurança em } \\
\text { centro cirúrgico: } \\
\text { validação de um } \\
\text { questionário para o } \\
\text { cenário brasileiro }\end{array}$ & $\begin{array}{l}\text { Lourenção,Tro } \\
\text { nchin. } 2018 \\
\text { (E3) }\end{array}$ & LILACS & Brasil & $\begin{array}{l}\text { Estudo de } \\
\text { Validação }\end{array}$ & $\begin{array}{l}590 \\
\text { Profissionais }\end{array}$ & 4 Meses & $\begin{array}{l}\text { Equipe } \\
\text { atuante no } \\
\text { C.C }\end{array}$ \\
\hline 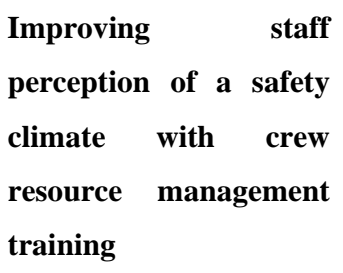 & $\begin{array}{l}\text { Kuy, et al. } \\
2017 \\
\text { (E4) }\end{array}$ & MEDLINE & EUA & Transversal & $\begin{array}{l}88 \\
\text { Profissionais }\end{array}$ & 12 Meses & $\begin{array}{l}\text { Equipe } \\
\text { atuante no } \\
\text { C.C }\end{array}$ \\
\hline $\begin{array}{l}\text { Cultura de segurança } \\
\text { entre os profissionais de } \\
\text { centro cirúrgico }\end{array}$ & $\begin{array}{l}\text { Cauduro et } \\
\text { al. } 2015 \\
\text { (E5) }\end{array}$ & BDENF & Brasil & Transversal & $\begin{array}{l}185 \\
\text { Profissionais }\end{array}$ & 3 Meses & $\begin{array}{l}\text { Equipe } \\
\text { atuante no } \\
\text { C.C e outros } \\
\text { profissionais } \\
\text { de saúde }\end{array}$ \\
\hline
\end{tabular}

Fonte: Autoras (2020).

Conforme o Quadro 2 é apresentado resultados e conclusões presentes nos artigos incluídos, de acordo com cada autor e sua percepção de cultura de segurança. 
Quadro 2. Caracterização da avaliação da cultura de segurança dos artigos incluídos. Recife, 2020.

\begin{tabular}{|c|c|c|}
\hline $\mathbf{N}$ & Resultados & Conclusão \\
\hline E1 & $\begin{array}{l}\text { A avaliação da segurança do paciente pelos profissionais foi } \\
\text { regular }(48,9 \%) \text {. A dimensão da cultura de segurança com } \\
\text { resultado mais positivo foi "Aprendizado organizacional- } \\
\text { melhoria contínua" }(58,7 \%) \text { e com resultados menos positivos } \\
\text { foram "Abertura para comunicação" (32,3\%) e "Feedback e } \\
\text { comunicação sobre erros" }(32,6 \%) \text {. }\end{array}$ & $\begin{array}{l}\text { Existem áreas problemáticas na cultura de segurança do } \\
\text { setor, mostrando que essa cultura precisa ser melhor } \\
\text { desenvolvida, com especial atenção às dimensões da } \\
\text { cultura que apresentaram avaliação menos positiva. }\end{array}$ \\
\hline E2 & $\begin{array}{l}\text { Enfermeiros que trabalham em salas de operação tiveram } \\
\text { pontuações mais baixas em comparação com áreas de } \\
\text { enfermaria e também relataram percepções negativas sobre a } \\
\text { gestão. Houve variação significativa nos escores de } \\
\text { comunicação em toda a especialidade clínica, mas nenhuma das } \\
\text { comparações alcançou significância estatística. A análise } \\
\text { temática identificou temas de fatores humanos, gestão clínica e } \\
\text { proteção de pacientes. }\end{array}$ & $\begin{array}{l}\text { Os resultados sugerem que o Questionário de Clima de } \\
\text { Segurança (SQC), utilizado como instrumento para a } \\
\text { mensuração do clima de segurança, requer mais } \\
\text { exploração. Os resultados indicam que a segurança na } \\
\text { prática de enfermagem é uma interação complexa entre os } \\
\text { sistemas de segurança e os aspectos sociais e interpessoais } \\
\text { da prática clínica. }\end{array}$ \\
\hline E3 & $\begin{array}{l}\text { A análise organizou um questionário com } 40 \text { assertivas alocadas } \\
\text { em seis domínios: clima de segurança, percepção da gerência, } \\
\text { percepção do estresse, condição do trabalho, comunicação no } \\
\text { ambiente cirúrgico e percepção do desempenho profissional. }\end{array}$ & $\begin{array}{l}\text { Esta pesquisa permitiu validar um instrumento passível de } \\
\text { avaliar o clima de segurança em centro cirúrgico. }\end{array}$ \\
\hline E4 & $\begin{array}{l}\text { Houve melhora em } 100 \% \text { das áreas avaliadas no questionário } \\
\text { de clima de segurança } 6 \text { meses após o treinamento sobre o } \\
\text { processo de construção de comunicação da equipe que visa } \\
\text { melhorar a segurança do paciente e do trabalho em equipe. Por } \\
1 \text { ano, essa melhoria foi sustentada em } 23 \text { das } 27 \text { áreas, com } \\
\text { destaque para as seguintes áreas: colaboração entre enfermeiros } \\
\text { e médicos e valorização da contribuição da enfermagem, } \\
\text { conhecimento sobre segurança do paciente e promoção } \\
\text { institucional de um clima de segurança do paciente. }\end{array}$ & $\begin{array}{l}\text { Os participantes relataram melhora em todos os } 27 \text { pontos } \\
\text { do questionário de clima de segurança em } 6 \text { meses em } \\
\text { comparação com a linha de base. }\end{array}$ \\
\hline E5 & $\begin{array}{l}\text { A baixa percepção da cultura de segurança foi evidenciada por } \\
\text { escores < } 75 \text { pontos entre } 73,5 \% \text { dos participantes. O escore } \\
\text { médio foi de } 62,7 \text { e entre os domínios variou de } 55,4 \text { para } \\
\text { Gerência da Unidade à } 76,9 \text { para Satisfação no Trabalho; } \\
\text { observou-se diferença estatística entre as categorias } \\
\text { profissionais em diversos aspectos da cultura de segurança. }\end{array}$ & $\begin{array}{l}\text { Os resultados apontam a necessidade de ações com vistas } \\
\text { a fortalecer a cultura de segurança do paciente } \\
\text { considerando a complexidade da assistência prestada em } \\
\text { centro cirúrgico. }\end{array}$ \\
\hline
\end{tabular}

Fonte: Autoras (2020).

\section{Discussão}

A Aliança Mundial para Segurança do Paciente, possui o objetivo de estimular os melhores cuidados aos pacientes durante o período de hospitalização, através do maior comprometimento dos profissionais para melhorar a segurança na assistência à saúde e assim elevar os índices na qualidade dos serviços prestados. Diante disso o Centro Cirúrgico (CC) com toda sua peculiaridade e riscos de erros e eventos adversos exige dos profissionais uma maior qualificação e maiores níveis de atenção (Hendges et al., 2020).

A cultura e o clima de segurança encontrados no centro cirúrgico, compõem os fenômenos organizacionais que são entendidos como: valores, crenças e normas que regem uma instituição. Dessa forma é importante deixar claro que, apesar de fazerem parte do mesmo fenômeno e, por vezes, serem utilizados como sinônimos, a cultura e o clima de segurança possuem conceitos distintos. As instituições que utilizam esses princípios necessitam estimular comunicação efetiva e medidas para o reconhecimento da importância da segurança (Santiago \& Turrini, 2015).

Diante disso, a cultura de segurança é definida como o produto de valores, padrões comportamentais individuais ou coletivos, que afirmam o compromisso e o estilo de gestão institucional. Enquanto o clima de segurança é definido como 
características mensuráveis da cultura organizacional, por meio de percepções e atitudes dos indivíduos, em determinado tempo. Assim sendo torna-se necessário a avaliação da cultura de segurança objetivando tanto o reconhecimento da situação organizacional como para averiguação do impacto de intervenções já realizadas (Costa et al., 2018).

A análise dos artigos mostrou que houve uma maior prevalência de enfermeiros entre os profissionais presentes nos estudos, porém a equipe atuante no CC também foi compreendida, dentre eles estavam: médicos cirurgiões e anestesistas, médicos residentes e de outras categorias como técnicos de farmácia e de laboratório, residentes e estagiários de enfermagem, biomédicos e fisioterapeutas. No ambiente cirúrgico a realização de uma assistência segura torna-se uma barreira para várias instituições de saúde. Os artigos selecionados relatam que cerca de 50\% dos eventos adversos que ocorrem poderiam ser evitados (Bezerra et al., 2015).

A cultura de adesão a segurança do paciente sempre se baseou em uma assistência livre de danos, tornando-se indispensável e necessária, diante disso a adesão pode ser caracterizada como a adoção de boas práticas visando a qualidade da assistência nos serviços de saúde, requisitando entendimento técnico do profissional, atitude ética e motivação. Sendo assim, nos estudos que foram analisados notou-se que todos os serviços adotavam os protocolos e estratégias da segurança do paciente, muito embora durante a execução tenha encontrado alguns obstáculos como: comportamento, estresse, condições do trabalho, convivência social e déficit de comunicação efetiva (Gutierres et al., 2019; Nascimento et al., 2015).

A comunicação foi identificada como um dos fatores que influenciam, pois, a falha na comunicação efetiva, reflete certa desarticulação das atividades entre a equipe multidisciplinar. Indicam o baixo diálogo, baixa interação e pouca troca de informação no ambiente de assistência cirúrgica. Interferindo diretamente, na assistência de qualidade e livre de riscos.

De acordo com dois dos estudos analisados, pode-se entender como comunicação efetiva comportamentos propositivos e favoráveis a transmitir, receber e interpretar informações com clareza, visando sempre o respeito mútuo. A busca por soluções para comportamentos que vão contra esses preceitos inclui: o uso de linguagem objetiva e caráter não-acusatório/punitivo que promovem comunicação e relações respeitosas, além de uma "via de abertura" para a realização de notificações, relatos e opiniões (Moreira et al., 2019).

Foi observada, a partir de um dos artigos analisados, a necessidade de reforçar a implementação das estratégias e ações de segurança, uma vez que, o processo de atendimento à saúde é favorável ao risco aumentado do paciente sofrer danos, ainda maior quando as normas e padrões estabelecidos na instituição são planejados ou executados de forma inadequada, dessa maneira torna-se indispensável a divulgação de informações que contribuam para a prevenção de danos ao paciente (Reis et al., 2016; Gomes et al., 2017).

Estudos ressaltam a importância do desenvolvimento de pesquisas na área de segurança do paciente como estratégia que fortalece os conhecimentos dos profissionais buscando-se assim, minimizar a ocorrência de eventos adversos através das mudanças de atitudes e incentivos a prática de uma cultura de segurança positiva, colaborando desta maneira com o processo do cuidar mais seguro nos serviços de saúde livre do medo de punições de caráter jurídico, ético e social (Batalha et al., 2015; Cavalcante et al., 2015).

Por fim, notou-se que todos os estudos contribuíram de uma forma satisfatória para a equipe de saúde que atua no CC, uma vez que auxiliam os profissionais a ter um maior domínio sobre o conceito do que é segurança do paciente, para assim identificar os fatores que influenciam de forma direta a segurança, bem como pontos positivos e negativos vivenciados diariamente e idealizar possíveis estratégias para melhorar as fragilidades (Marique et al., 2015).

\section{Conclusão}

A cultura de segurança encontrada no $\mathrm{CC}$, contribuiu positivamente para as equipes atuantes e os demais profissionais 
de saúde, porém evidenciou-se que ainda possui obstáculos na prática, na qual é necessária uma força conjunta entre a gerência e os profissionais atuantes nos cenários de assistência ao paciente para aperfeiçoamento e incorporação dessa prática no serviço.

Visto que, a avaliação da cultura de segurança permite a identificação das vulnerabilidades encontradas no serviço, e consequentemente, os resultados contribuem para o planejamento de intervenções coerentes com as necessidades ou fragilidades identificadas.

A segurança do paciente tornou-se uma temática prioritária na área da saúde, devido aos inúmeros incidentes e a sua relevância para o serviço de saúde. Por isso, as instituições de saúde cada vez mais vem utilizando a segurança do paciente como estratégia para garantir uma assistência de qualidade, com ausência de erros e eventos adversos.

Diante disso destaca-se a importância da ampliação do conhecimento sobre a segurança do paciente e a cultura de segurança, visando produzir um impacto positivo na qualidade da assistência e nas boas práticas em saúde.

\section{Referências}

Batalha, E. M. S. S. \&, Melleiro M. M. (2016). Cultura de segurança do paciente: percepções da equipe de enfermagem. Hu Revista, 42(2): $133-142$.

Bezerra, W. R., Bezerra, A. L. Q., Paranaguá, T. T. B., Bernardes, M. J. C., \& Teixeira, C. C. (2015). Ocorrência de incidentes em um centro cirúrgico: estudo documental. Revista Eletrônica de Enfermagem, 17(4) 1-11.

Brasil. Ministério da Saúde. (2014). Documento de referência para o programa nacional de segurança do paciente. Ministério da Saúde. https://bvsms.saude.gov.br/bvs/publicacoes/documento_referencia_programa_nacional_seguranca.pdf.

Cavalcante, A. K. C. B., Rocha, R. C., Nogueira, L. T., Avelino, F. V. S. D., \& Rocha, S. S. (2015). Cuidado seguro ao paciente: contribuições da enfermagem. Revista Cubana de Enfermería, 31(4): 0-0.

Costa, D. B., Ramos, D., Gabriel, C. S., \& Bernardes, A. (2018). Cultura De Segurança Do Paciente: Avaliação Pelos Profissionais De Enfermagem. Texto Contexto-Enfermagem, 27(3): e2670016.

Dezordi, C. C. M., Stumm, E. M. F. (2018). Security attitudes of a team before and after safe surgery checklist implementation. Journal of Nursing UFPE Online, $12(3): 816-9$.

Gomes, A. T. L., Salvador, P. T. C. O., Rodrigues, C. C. F. M., Silva, M. F., Ferreira, L. L., \& Santos, V. E. P. (2017). A segurança do paciente nos caminhos percorridos pela enfermagem brasileira. Revista Brasileira De Enfermagem, 70(1): 146-154.

Gutierres, L. S., Santos, J. L. G., Barbosa, S. F. F., Maia, A. R. C., Koerich, C., \& Gonçalves, N. (2019). Adesão aos objetivos do Programa Cirurgias Seguras Salvam Vidas: Perspectiva de enfermeiros. Revista Latino-Americana de Enfermagem, 27: e310827.

Heidmann, A., Trindade, L. F., Schmid, C. R., Loro, M. M., Fontana, R. T., \& Kolankiewicz, A. C. B. (2019). Fatores contribuintes para consolidação da cultura de segurança do paciente no âmbito hospitalar. Escola Anna Nery, 24(1): e20190153.

Hendges, M., Soares, N. V., Rodrigues, F. C. P., \& Bittencourt, V. L. B. (2020). Checklist Cirúrgico e Sua Importância Na Segurança Do Paciente. Revista Vivências, 16(31): 245-252.

Henriques, A. H. B., Costa, S. S., \& Lacerda, J. S. (2016). Assistência De Enfermagem Na Segurança Do Paciente Cirúrgico: Revisão Integrativa. Cogitare Enfermagem, 21(4): 1-9.

Manrique, B. T., Soler, L. M., Bonmati, A. N., Montesinos, M. J. L., \& Roche, F. P. (2015). Segurança do paciente no centro cirúrgico e qualidade documental relacionadas à infecção cirúrgica e à hospitalização. Acta Paulista De Enfermagem, 28(4): 355-60.

Moreira, F. T. L. S., Callou, R. C. M., Albuquerque, G. A., \& Oliveira, R. M. (2019). Estratégias de comunicação efetiva no gerenciamento de comportamentos destrutivos e promoção da segurança do paciente. Revista Gaúcha de Enfermagem, 40(esp): e20180308.

Nascimento, J. C., \& Draganov P. B. (2015). História da qualidade em segurança do paciente. História Da Enfermagem: Revista Eletrônica, 6(2): 299-309.

Reis, G. A. X., Hayakawa, L. Y., Murassaki, A. C. Y., Matsuda, L. M., Gabriel, C. S., \& Oliveira, M. L. F. (2017). Implantação Das Estratégias De Segurança Do Paciente: Sugestões De Enfermeiros Gestores. Texto Contexto Enfermagem, 26(2): e00340016.

Roscani, A. N. C. P., Ferraz, E. M., Filho, A. G. O., \& Freitas, M. I. P. (2015). Validação de checklist cirúrgico para prevenção de infecção de sítio cirúrgico. Acta Paulista De Enfermagem, 28(6): 553-565.

Sanchis, D., Haddad, M. C. F. L., Girotto, E., Silva, A. M. R. (2020). Cultura de segurança do paciente: percepção de profissionais de enfermagem em instituições de alta complexidade. Revista Brasileira De Enfermagem REBEn, 73(5): e20190174.

Santiago, T. H. R., \& Turrini, R. N. T. (2015). Cultura e clima organizacional para segurança do paciente em unidades de terapia intensiva. Revista Da Escola De Enfermagem Da Usp, 49(Esp): 123-130. 
Research, Society and Development, v. 10, n. 9, e53710918175, 2021

(CC BY 4.0) | ISSN 2525-3409 | DOI: http://dx.doi.org/10.33448/rsd-v10i9.18175

Serra, J. N., Barbiere, A. R., \& Cheade, M. F. M. (2016). Situação dos hospitais de referência para implantação/funcionamento do núcleo de segurança do paciente. Cogitare Enfermagem, 21 n. esp: 01-9.

Wegner, W., Silva, S. C., Kantorski, K. J. C., Predebon, C. M., Sanches, M. O., Pedro, E. N. R. (2016). Educação Para Cultura Da Segurança Do Paciente: Implicações Para A Formação Profissional. Escola Anna Nery, 20(3): e20160068. 